\title{
Metallo- $\beta$-lactamase producing Enterobacter cloacae: An emerging threat in neonates
}

\author{
Muhammad Usman Qamar*, Abdul Hannan, Muhammad Usman Arshad and \\ Muhammad Arshad
}

Department of Microbiology, University of Health Sciences, Lahore, Pakistan.

Accepted 13 January, 2014

\begin{abstract}
Enterobacter cloacae is not a primary human pathogen but has obtained an important role as a nosocomial pathogen. Acquiring this property of metallo- $\beta$-lactamases (MBL) producers, the pathogen becomes very virulent for the susceptible neonates and leaves little choice of antibiotics for its management. Blood samples $(n=103)$ were collected in peadriatic blood culture bottles from suspected neonates aseptically. The samples were processed in BACTEC 9120. Antimicrobial susceptibility testing was performed using Kirby-Bauer disc diffusion method according to CLSI 2009. Carbapenems resistant E. cloacae were further analyzed for the detection of carbapenamase as per CLSI guidelines and MBL production by double disc potentiation method. Of 103 blood cultures specimens, 71 were declared positive by BACTEC 9120 and 91 isolates were recovered. Among these isolates, $E$. cloacae $(n=12)$ were found to be carbapenems resistant and 10 out of these were carbapenamase and MBL producers. All $M B L$ producing pathogens were pan-drug resistant. The spread of MBL among $E$. cloacae is an emerging threat particularly in hospitalized neonates.
\end{abstract}

Key words: Enterobacter cloacae, metallo- $\beta$-lactamases (MBL), antimicrobial resistance.

\section{INTRODUCTION}

Enterobacter cloacae are facultative Gram-negative bacilli which primarily are not the human pathogens, widely distributed in the environment and normal flora of gastrointestinal tract of 40 to $80 \%$ of humans (Keller et al., 1998). But over the last 20 years, it has become an important opportunistic pathogen in hospitalized patients particularly among neonates (Gbaguidi-Haore et al., 2008). According to Surveillance and Control of Pathogens of Epidemiological Importance (SCOPE) survey report of USA, Enterobacter spp. (mainly E. cloacae) accounted for $3.9 \%$ of all nosocomial bloodstream infections ranking eighth among all microorganisms causing bacteraemia (Wang et al., 2009). Mortality rate caused by Enterobacter spp. ranges from 15 to $87 \%$ annually throughout the world (Wagenlehner et al., 2002). E. cloacae are also responsible for outbreaks in neonatal units and major risk factors are contaminated door handles, pharmaceutical products; cross contami- nation by healthcare worker hands, medical devices and equipments (Van Den Berg et al., 2000; Dijk et al., 2002). The pathogenicity of $E$. cloacae has been increased because of intrinsic resistance to ampicillin and narrowspectrum cephalosporins and exhibits a high frequency of mutation to resistance to expanded-spectrum cephalosporins and carbapenems. They also possess different types of $\beta$-lactamases producing enzymes including metallo- $\beta$-lactamases (MBLs). These enzymes (MBLs) belongs to ambler class $B$ that require divalent cations as cofactor for their activity which are inhibited by the action of metal ions chelator such as ethylene diamine tetraacetic acid (EDTA) (Lee et al., 2008). They have potent hydrolyzing activity not only against carbapenems but also against other betalactam antibiotics. These are not inhibited by the $\beta$-lactamase inhibitors like clavulanic acid, sulbactam and tazobactam (Butt et al., 2005; 
Table 1. Total number of carbapenem resistant, carbapenamase and MBL producer E. cloacae.

\begin{tabular}{lc}
\hline Enterobacter cloacae & Number \\
\hline Carbapenems resistant E. cloacae & 12 \\
Carbapenamase and MBL producer & 10 \\
\hline
\end{tabular}

Chen et al., 2009). In recent years, MBL producing microorganisms have become a major cause of nosocomial infections and out breaks throughout the world (Maltezou, 2009). MBL have been reported mainly in $P$. aeruginosa and Acinetobacter spp. but recent reports have also documented their presence in the family of Enterobacteriaceae (Gacar et al., 2005; Siarkou et al., 2009). The spread of $\mathrm{MBL}$ genes among $E$. cloacae is a matter of great concern particularly in hospitalized patients (Falcone et al., 2009). To our knowledge, there is only one case report of $\mathrm{MBL}$ producing $P$. aeruginosa in Pakistan (Butt et al., 2005) and none have been reported in Enterobacteriaceae.

The objective of the study was to investigate the presence of MBL among carbapenem resistant isolates of E. cloacae.

\section{MATERIALS AND METHODS}

Prior to the start of the study, permission was taken from ethical review committee, University of Health Sciences, Lahore, Pakistan.

\section{Samples size}

It was an observational study. A total of 103 neonates suspected of septicemia were included and blood samples were collected from a neonatology unit (50 bedded) of tertiary care hospital Lahore, Pakistan during April to September, 2009.

\section{Blood cultures}

Blood collection was performed by aseptic technique. Pediatric blood culture bottles (Bactec Peds plus/F) were inoculated with 1-3 $\mathrm{ml}$ of blood and incubated in BACTEC 9120 instrument (Becton Dickinson, USA) for at least 5 days before declaring negative.

\section{Identification/purification of isolates}

Positive blood culture $(n=71)$ specimens were sub-cultured on blood and MacConkey agar and incubated at $35^{\circ} \mathrm{C}$ for $24 \mathrm{~h}$. Out of positive cultures, 91 organisms were isolated. Among these isolates E. cloacae $(n=12)$ were identified on the basis of morphology, culture characteristics and API 20E (BioMerieux France).

\section{Antimicrobial susceptibility testing}

Antimicrobial susceptibility of isolates was performed by KirbyBauer disk diffusion method using Mueller-Hinton agar (Oxoid UK). The plates were prepared and incubated at $35^{\circ} \mathrm{C}$ for $24 \mathrm{~h}$. Implanted antibiotics were ampicillin $(10 \mu \mathrm{g})$, co-amoxiclav $(20 \mu \mathrm{g} / 10 \mu \mathrm{g})$, cefuroxime $(30 \mu \mathrm{g})$, ceftriaxone $(30 \mu \mathrm{g})$, ceftazidime $(30 \mu \mathrm{g})$, cefepime
(30 $\mu \mathrm{g})$, ciprofloxacin $(5 \mu \mathrm{g})$, amikacin $(30 \mu \mathrm{g})$, sulfamethoxazole/trimethoprim $(1.25 / 23.75 \mu \mathrm{g})$, imipenem $(10 \mu \mathrm{g})$, and meropenem $(10 \mu \mathrm{g})$. The interpretation of susceptibility results were done according to Clinical Laboratory Standards Institute (CLSI) 2009 guidelines (Wikler et al., 2009).

\section{Carbapenamase and MBL detection}

E. cloacae found to be resistant to imipenem and meropenem were further tested for carbapenamase and MBL production. Carbapenamase production was detected by Modified Hodge's Test (MHT) as per CLSI 2009 guidelines. Carbapenamase production is detected by the MHT when the test organism produces the enzyme and allows growth of carbapenems susceptible strain ( $E$. coli ATCC 25922) towards a carbapenem disc. The result is characteristic cloverleaf-like indentations.

MBL detection was done by the double disc potentiation method using inhibitor ethylene diamine tetra amino acetic acid (EDTA). Results were interpreted as positive when an increase in zone size of at least $7 \mathrm{~mm}$ around the imipenem-EDTA disc and meropenemEDTA disc was observed (Varaiya et al., 2008). Positive and negative controls were run parallel.

\section{RESULTS}

Of 103 blood cultures specimens, 71 were declared positive by BACTEC 9120 and 91 isolates were recovered. Among these isolates $E$. cloacae $(n=12)$ were found to be carbapenems resistant and $10(83 \%)$ out of these were carbapenamase and $\mathrm{MBL}$ producers as shown in Table 1. Carbapenamase production was confirmed using MHT (Figure 1). Carbapenemase producing isolates were further identified for MBL by double disc potentiation method (Figure 2). Antimicrobial susceptibility pattern of carbapenamase and MBL producing $E$. cloacae is shown in Table 2. MBL producing E. cloacae $(n=10)$ were found to be pan-drug resistant.

\section{DISCUSSION}

E. cloacae are recognized as an emerging nosocomial pathogen particularly in intensive care units. These are intrinsically resistant to older antibiotics and have ability to produce resistance against newer drugs (carbapenems) as well (Wang et al., 2009; Paauw et al., 2008; Sanders et al., 1997). Due to the changing bacteriological profile, new treatment alternatives are being explored throughout the world (Mahapatra et al., 2002). High degree of carbapenems resistance E. cloacae $(n=12)$ was detected in this study (Table 1). Among these carbapenems resistant pathogens, E. cloacae $(n=10)$ were carbapenamase and MBL producers (Table 2). IMI-1 type of MBL was isolated in California, USA from two isolates of E. cloacae in 1984 (Rasmussen et al., 1996). Whereas, in China, plasmid mediated IMI-2 type MBL producing isolate of $E$. cloacae was found in 2001 (Mahapatra et al., 2002).

In the present study, pan-drug resistance of E. cloacae 


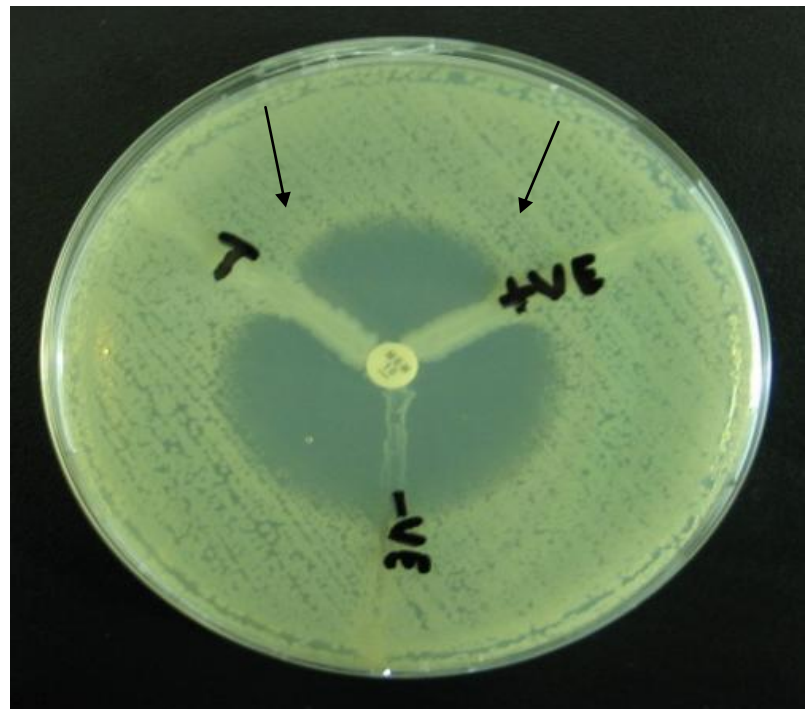

Figure 1. Demonstration of MHT. The MHT plate shown contains a lawn of carbapenem susceptible E. coli (ATCC 25922). A meropenem $(10 \mu \mathrm{g})$ disc was placed in the center of plate. Positive control (E. cloacae), negative control (Sphingomonas paucimobilis) and test organism has been streaked from the edge of disc to the edge of the plate. Arrows indicate the clover-leaf like indentation indicating the production of carbapenem.

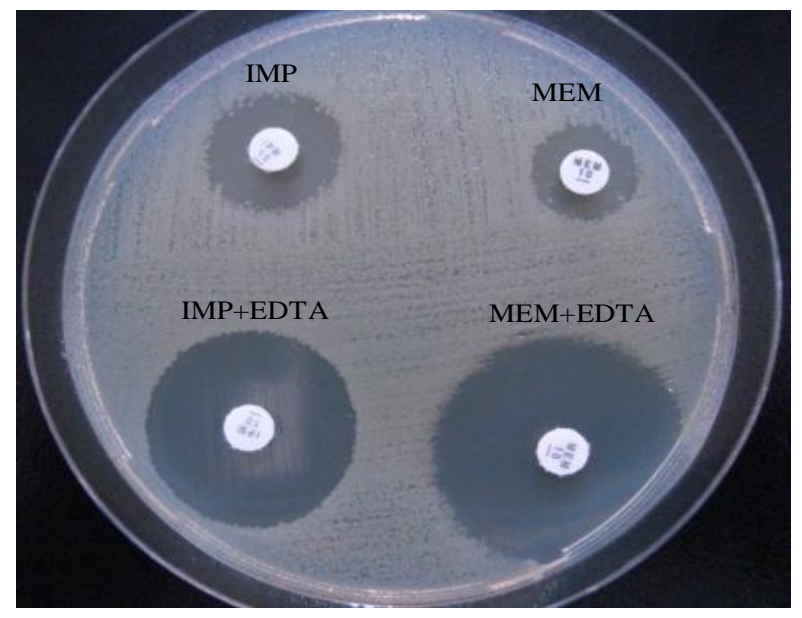

Figure 2. Double disc potentiation test. The test organism was inoculated on the Mueller-Hinton agar plate. Two imipenem discs $(10 \mu \mathrm{g})$ and two meropenem discs $(10 \mu \mathrm{g})$ were placed. $0.5 \mathrm{M}$ EDTA solution was added to one imipenem and one meropenem disc. Increase in zone size $(>7 \mathrm{~mm})$ in EDTA containing discs represents the MBL production.

was observed (Table 2). An Indian study on neonatal septicemia reported four cases of Acinetobacter spp. that were resistant to imipenem. Among these resistant pathogens only one Acinetobacterspp. was MBL producer (Arora et al., 2006). According to our knowledge, isolation
Table 2. Antimicrobial resistance patterns of carbapenamase and MBL producing E. cloacae $(n=10)$.

\begin{tabular}{lc}
\hline Antimicrobial & Resistance pattern \\
\hline Ampicillin & $\mathrm{R}$ \\
Co-amoxiclav & $\mathrm{R}$ \\
Cefuroxime & $\mathrm{R}$ \\
Ceftriaxone & $\mathrm{R}$ \\
Ceftazidime & $\mathrm{R}$ \\
Cefepime & $\mathrm{R}$ \\
Amikacin & $\mathrm{R}$ \\
Ciprofloxacin & $\mathrm{R}$ \\
Co-trimoxazole & $\mathrm{R}$ \\
Imipenem & $\mathrm{R}$ \\
Meropenem & $\mathrm{R}$ \\
\hline
\end{tabular}

of $E$. cloacae in neonatal septicemia has not been reported at national level so far. This alarming increase in the rate of multidrug resistance is mainly linked to poor infection-control practices that lead to out-breaks of infections and person to person transmission of resistant pathogens. For instance, common identified sources in our setup for these resistant bugs are contaminated intravenous catheter, feeding tubes, various environmental surfaces and colonized hands of staff.

Nurseries are often seriously overcrowded and understaffed, sharing of baby beds (two to three babies in a cot). Substandard sterilization and disinfection practices are common. It is well documented that neonates have immature immune system and unable to provide defense against virulent pathogens. Premature babies are at high risk because of lack of protective maternal antibodies, underdeveloped innate immunity and fragile, easily damaged skin (Hannan et al., 2013). Another major factor to acquire resistance in our setup is irrational use of empirical therapy which is not according to the WHO criteria (WHO, 2002).

\section{Conclusion}

The spread of MBL among E. cloacae is an alarming threat particularly in hospitalized neonates. Metallo- $\beta$-lactamase producing microorganisms being the most resistant ones must be contained inside the hospital environments. A national multicenter survey should also be carried to have a fair idea of resistance pattern and devise proper antibiotic policy to combat this serious problem.

\section{ACKNOWLEDGEMENTS}

We would like to thank the head and staff of Neonatology Department of Tertiary Care Hospital Lahore, for their kind support. 


\section{REFERENCES}

Arora U, Jaitwani J (2006). Acinetobacter spp. - an emerging pathogen in neonatal septicemia in Amritsar. Indian J. Med. Microbiol. 24(1):81.

Butt T, Usman M, Ahmad RN, Saif I (2005). Emergence of metallo-betalactamase producing Pseudomonas aeruginosa in Pakistan. J. Pak. Med. Assoc. 55(7):302-304.

Chen LR, Zhou HW, Cai JC, Zhang R, Chen GX (2009). Detection of plasmid-mediated IMP-1 metallo-beta-lactamase and quinolone resistance determinants in an ertapenem-resistant Enterobacter cloacae isolate. J. Zhejiang Univ. Sci. B. 10(5):348-354.

Dijk Yv, Bik EM, Hochstenbach-Vernooij S, Vlist GJvd, Savelkoul PHM, Kaan JA (2002). Management of an outbreak of Enterobacter cloacae in a neonatal unit using simple preventive measures. J. Hosp. Infect. 51(1):21-26.

Falcone M, Mezzatesta ML, Perilli M, Forcella C, Giordano A, Cafiso V, Amicosante G, Stefani S, Venditti M (2009). Infections with VIM-1 Metallo- $\beta$-Lactamase-Producing Enterobacter cloacae and Their Correlation with Clinical Outcome. J. Clin. Microbiol. 47(11):35143519.

Gacar GG, Midilli K, Kolayli F, Ergen K, Gundes S, Hosoglu S (2005). Genetic and enzymatic properties of metallo-beta-lactamase VIM-5 from a clinical isolate of Enterobacter cloacae. Antimicrob. Agents Chemother. 49(10):4400-3.

Gbaguidi-Haore $H$, Talon $D$, Thouverez $M$, Menget $A$, Bertrand $X$ (2008). Molecular epidemiology of Enterobacter cloacae in a neonatal department: a 2-year surveillance study. Eur. J. Clin. Microbiol. Infect. Dis. 27(8):643-648.

Hannan A, Qamar MU, Usman M, Waheed KA, Rauf K (2013). Multidrug resistant microorganisms causing neonatal septicemia: In a tertiary care hospital Lahore, Pakistan. Afr. J. Microbiol. Res. 7(19):1896-1902.

Keller R, Pedroso MZ, Ritchmann R, Silva RM (1998). Occurrence of virulence-associated properties in Enterobacter cloacae. Infect. Immun. 66(2):645-649.

Lee MF, Peng CF, Hsu HJ, Chen YH (2008). Molecular characteris ation of the metallo-beta-lactamase genes in imipenem-resistant Gramnegative bacteria from a university hospital in southern Taiwan. Int. J. Antimicrob. Agents. 32(6):475-480.

Mahapatra A, Ghosh S, Mishra S, Pattnaik D, Pattnaik K, Mohanty S (2002). First isolation of bla IMI-2 in an Enterobacter cloacae clinical isolate from China. Indian J. Med. Microbiol. 20(2):110-112.

Mahapatra A, Ghosh SK, Mishra S, Pattnaik D, Pattnaik K, Mohanty SK (2002). Enterobacter cloacae: a predominant pathogen in neonatal septicaemia. Indian J. Med. Microbiol. 20(2):110-112.

Maltezou HC (2009). Metallo-beta-lactamases in Gram-negative bacteria: introducing the eraof pan-resistance? Int. J. Antimicrob. Agents 33(5):405.e1-7.
Paauw A, Caspers MP, Schuren FH, Leverstein-van Hall MA, Deletoile A, Montijn RC (2008). Genomic diversity within the Enterobacter cloacae complex. PLoS One 3(8):3018.

Rasmussen BA, Bush K, Keeney D, Yang Y, Hare R, O'Gara C (1996). Characterization of IMI-1 beta-lactamase, a class A carbapenemhydrolyzing enzyme from Enterobacter cloacae. Antimicrob. Agents Chemother. 40(9):2080-6.

Sanders WE, Sanders CC (1997). Enterobacter spp.: pathogens poised to flourish at the turn of the century. Clin. Microbiol. Rev. 10(2):22041.

Siarkou VI, Vitti D, Protonotariou E, Ikonomidis A, Sofianou D (2009). Molecular epidemiology of outbreak-related pseudomonas aeruginosa strains carrying the novel variant blaVIM-17 metallo-betalactamase gene. Antimicrob. Agents Chemother. 53(4):1325-30.

Van Den Berg RW, Claahsen HL, Niessen M, Muytjens HL, Liem K, Voss A (2000). Enterobacter cloacae outbreak in the NICU related to disinfected thermometers. J. Hosp. Infect. 45(1):29-34.

Varaiya A, Kulkarni N, Kulkarni M, Bhalekar P, Dogra J (2008). Incidence of metallo beta lactamase producing Pseudomonas aeruginosa in ICU patients. Indian J. Med. Res. 127(4):398-402.

Wagenlehner FM, MacKenzie FM, Forbes KJ, Gould IM (2002). Molecular epidemiology and antibiotic resistance of Enterobacter spp. from three distinct populations in Grampian, UK. Int. J. Antimicrob. Agents 20(6):419-425.

Wang R, Wu S, Li X, He P, Liu Y (2009). Detection of AmpC $\beta$ lactamase and drug resistance of Enterobacter cloacae. Front. Med. China 3(1):72-75.

WHO (2002). Explore simplified antimicrobial regimens for the treatment of neonatal sepsis. meeting report. Department of child and adolescent health anddevelopment Geneva.

Wikler MA, Cockerill FR, Bush K, Dudley MN, Eliopoulos GM (2009). Performance Standards for Antimicrobial Susceptibility Testing; Nineteenth International Supplement. 29(3). 